\title{
Human Papillomavirus Coinfection in the Cervical Intraepithelial Lesions and Cancer of Mexican Patients
}

\author{
Juan Ramón Padilla-Mendoza (iD, ${ }^{1}$ Lucía Angélica Gómez-López $\mathbb{D}^{\mathrm{D}}{ }^{2}$ \\ Mavil López-Casamichana ${ }^{D},{ }^{2}$ Elisa Irene Azuara-Liceaga ${ }^{(D)}{ }^{2}$ \\ Enoc Mariano Cortés-Malagón $\left(\mathbb{D},{ }^{3}\right.$ Lilia López-Cánovas $\mathbb{D}^{2}{ }^{2}$ \\ Octavio Daniel Reyes-Hernández $\mathbb{D}^{4},{ }^{4}$ Mario Alberto Rodríguez $\mathbb{D}^{1},{ }^{1}$ José Bonilla-Delgado $\mathbb{D},{ }^{3}$ \\ and Israel López-Reyes $\mathbb{D D}^{5}$
}

\author{
${ }^{1}$ Departamento de Infectómica y Patogénesis Molecular, Centro de Investigación y de Estudios Avanzados (CINVESTAV-IPN), \\ Ciudad de México, Mexico \\ ${ }^{2}$ Posgrado en Ciencias Genómicas, Universidad Autónoma de la Ciudad de México (UACM), Plantel Del Valle, \\ Ciudad de México, Mexico \\ ${ }^{3}$ Unidad de Genética y Cáncer, Hospital Juárez de México (HJM), Ciudad de México, Mexico \\ ${ }^{4}$ Facultad de Estudios Superiores Zaragoza, Universidad Nacional Autónoma de México (UNAM), Ciudad de México, Mexico \\ ${ }^{5}$ Universidad Autónoma de la Ciudad de México (UACM), Plantel Cuautepec, Ciudad de México, Mexico
}

Correspondence should be addressed to Israel López-Reyes; israel.lopez.reyes@uacm.edu.mx

Received 7 August 2020; Revised 20 October 2020; Accepted 23 October 2020; Published 16 November 2020

Academic Editor: Giuliana Banche

Copyright (c) 2020 Juan Ramón Padilla-Mendoza et al. This is an open access article distributed under the Creative Commons Attribution License, which permits unrestricted use, distribution, and reproduction in any medium, provided the original work is properly cited.

\begin{abstract}
According to their oncogenic properties, Human Papillomaviruses (HPVs) are classified into two types: Low-Risk (LR-HPVs) and High-Risk Human Papillomaviruses (HR-HPVs). The immune system naturally controls the majority of HPV infections; however, when the HR-HPV infection is persistent, the risk of developing cervical cancer increases. Previous studies indicate that multipleinfection or coinfection with HR-HPV occurs frequently and can potentiate the development of cervical lesions. This study aimed to establish the HPV coinfection rate in squamous intraepithelial lesions from Mexican patients. For HPV detection, we performed PCR on 55 cervical lesions diagnosed by colposcopy. We detected the presence of HPV infection in $87.27 \%$ (48/55) of the lesions; interestingly, HPV coinfection was observed in 70.83\% (34/48) of these samples. We also evaluated HPV infection in adjacent areas without morphological changes from 25 samples. The results showed that 80\% (20/25) of these were HPV-positive and, curiously, all presented HPV-16 infection. In conclusion, our results revealed a high prevalence of HPV coinfection in cervical lesions in Mexican patients, and these results contribute to future research focused on the role that HPV coinfection plays in the development of cervical cancer.
\end{abstract}

\section{Introduction}

Cervical cancer (CC) is the second type of cancer among women in developing countries and is the primary neoplasm associated with Human Papillomavirus (HPV) infection [1]. Late detection of CC and its precursor lesions can lead to the death of these women, making it a public health problem [2]. Persistent infection in the cervix with High-Risk Human
Papillomaviruses (HR-HPV) produces precancerous lesions that eventually progress to CC [3-5].

There are more than $200 \mathrm{HPV}$ types, of which 12-15 are classified as HR-HPV [6, 7]. Cervical infection with more than one HPV genotype (coinfection) is common in 20$50 \%$ of HPV-infected women, especially among young women [8]. Infection with multiple HPV types is often considered a risk factor leading to the development of CC [9]. 
However, the relationship between multiple HPV-type infections and the progression of CC is questionable and remains unclear. The main debate lies in whether these infections occur due to affinity among certain HPV types or randomly. The establishment of persistent HPV infection is challenging; although, coinfection is probably involved, perhaps by means of competitive or cooperative interactions between HPV types, which could increase or decrease the risk of the progression of CC. Despite several contradictions, few studies have evaluated the relationship between multiple HPV-type infections and the risk of cervical disease [10].

In this study, we analyzed multiple HPV types of infection in biopsies from Mexican patients diagnosed with cervical lesions and CC. Our data revealed the presence of multiple HPV-type infections in the majority of samples with lesion $(70.83 \%)$, but only $30 \%$ in adjacent tissue without morphological changes.

\section{Materials and Methods}

2.1. Samples. Biopsies were obtained from Mexican women seen at Hospital IMSS "La Raza" (Mexico City) and "Hospital Juárez de México" (Mexico City) and who were pathologically diagnosed with Atypical Squamous Cells of Undetermined Significance (ASCUS), Low-grade Squamous Intraepithelial Lesion (LSIL), High-grade Squamous Intraepithelial Lesion (HSIL), or Cervical Cancer (CC). The present study conducted according to the Declaration of Helsinki for Medical Protocol and Ethics, and the Institutional Committee of Research and Ethics approved the study (registration no. HMJ 2231/13-B). Expert colposcopists performed all examinations, and samples were classified according to the International Federation of Gynecology and Obstetrics (FIGO) [11]. Fifty-five biopsies of the cervical lesions from patients with an age range between 20 and 65 years (8 ASCUS, 25 LSIL, 11 HSIL, and 11 CC) plus 25 biopsies of adjacent regions without morphological changes, for a total of eighty samples, were analyzed.

Biopsies were placed in Tissue-Tek medium (Sakura Finetek, USA) for their preservation and stored at $-80^{\circ} \mathrm{C}$ prior to DNA extraction.

2.2. HPV Detection and Genotyping. Samples were mechanically disrupted and subsequently homogenized in lysis buffer for DNA extraction according to the established protocol by the DNA/RNA All Prep Kit (Qiagen ${ }^{\circledR}$, USA). Amplification of the gapdh gene was performed to test sample sufficiency employing the template $100 \mathrm{ng}$ of DNA and targeting a 140-bp sequence with the following primers: forward: $5^{\prime}$ -AGGTGACACTATAGAATAACCGTCAAGGC-3'; ; and reverse: $\quad 5^{\prime}$-GTACGHACTCACTATAGGGATGGTGGT GA-3'. To detect HPV, we performed PCR assays using the PGMY09/PGMY11 primers (MY11: 5'-GCMCAGG GWCATAAYAATGG-3'; MY09: $5^{\prime}$-CGTCCMARRGGAWACTGATC- $3^{\prime}-$ ) targeting a 450 -bp sequence of the $L 1$ gene [12]. According to the established protocol, genotyping was performed using the Kit CLART ${ }^{\circledR} \mathrm{HPV} 2$ (Genomica ${ }^{\circledR}$, Spain). This kit, through multiplex PCR and low density arrays, detects the following: $35 \mathrm{HPV}$ types, including 14 high-risk (HR-HPV) (HPV-16, HPV-18, HPV-31, HPV-33, HPV-35, HPV-39, HPV-45, HPV-51, HPV-52, HPV-56, HPV-58, HPV-59, HPV-66, and HPV-68B), four probably High-Risk (HPV-26, HPV-53, HPV-73, and HPV-82), and 17 Low-Risk (LR-HPV) (HPV-6, HPV-11, HPV-40, HPV42, HPV-43, HPV-44, HPV-54, HPV-61, HPV-62, HPV70, HPV-71, HPV-72, HPV-81, HPV-83, HPV-84, HPV85, and HPV-89).

2.3. Statistical Analysis. A woman (biopsy) infected with more than one HPV genotype was considered to have a multiple HPV-type infection or coinfection. To determine Student's $t$-test independence among variables, analysis of variance (ANOVA) was utilized to compare groups. Odds ratios (OR), calculated with the Medcalc ${ }^{\circledR}$ and StatSoft $8.0^{\circledR}$ software, were determined to estimate the association of genotypes with cervical carcinoma. $P$ values of $<0.05$ were considered statistically significant.

\section{Results}

This study included biopsies from 55 patients with a mean age of 42.5 years. Samples were grouped according to their pathological diagnosis. Thus, we analyzed eight biopsies diagnosed with ASCUS (15\%), 25 biopsies diagnosed with LSIL (45\%), 11 biopsies diagnosed with HSIL (20\%), and 11 biopsies diagnosed with CC (20\%). Moreover, from 25 of these biopsies (6 with LSILs, 10 with HSILs, and 9 with CCs), we obtained a sample of adjacent tissue without morphological changes.

Overall, 48 of 55 biopsies with cervical lesions were HPV positive; therefore, HPV prevalence was $87.27 \% ; 95.83 \%$ (46/48) of HPV-positive biopsies contained HR-HPV (Figure 1(a)) corresponding to 13 genotypes, among which the most frequent were HPV-16 (52.08\%), HPV-31 (22.91\%), HPV-51 (18.75\%), and HPV-18 (16.66\%) (Figure 1(b)). On the other hand, 43.75\% (21/48) of the HPV-positive samples presented LR-HPV (Figure 1(a)) with 12 different genotypes, among which the most frequent were HPV-61, HPV-70, and HPV-84 (6.25\% for each genotype) (Figure 1(b)).

Interestingly, we found that $70.83 \%$ (34/48) of HPVpositive samples presented more than one HPV genotype (coinfection), while the remained only had a single infection (Table 1). Of the samples with coinfection, 55.88\% (19/34) demonstrated a mixed coinfection (infection with HR- and LR-HPV). The remaining 44.12\% (15/34) displayed a simple coinfection (genotypes of the same HPV group), of which 13 (38.23\%) were coinfection with HR-HPV genotypes, whereas only two (5.88\%) exhibited coinfection with LR-HPV genotypes (Table 1). HPV-31 and HPV-51 were the most common genotypes in LSIL, while HPV-16 was the main genotype in CC (data not shown).

In that, the most frequent HR-HPVs in CC at worldwide are HPV-16 and HPV-18 [13]; we evaluated the combination of these genotypes with other HR-HPV genotypes. Of the 13 samples with HR-HPV simple coinfection, seven (53.84\%) showed the presence of HPV-16, whereas coinfection of 


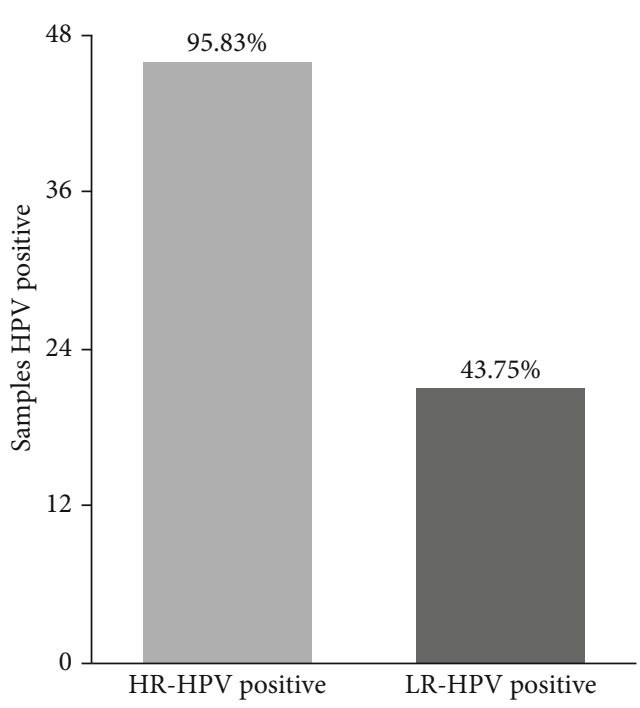

(a)

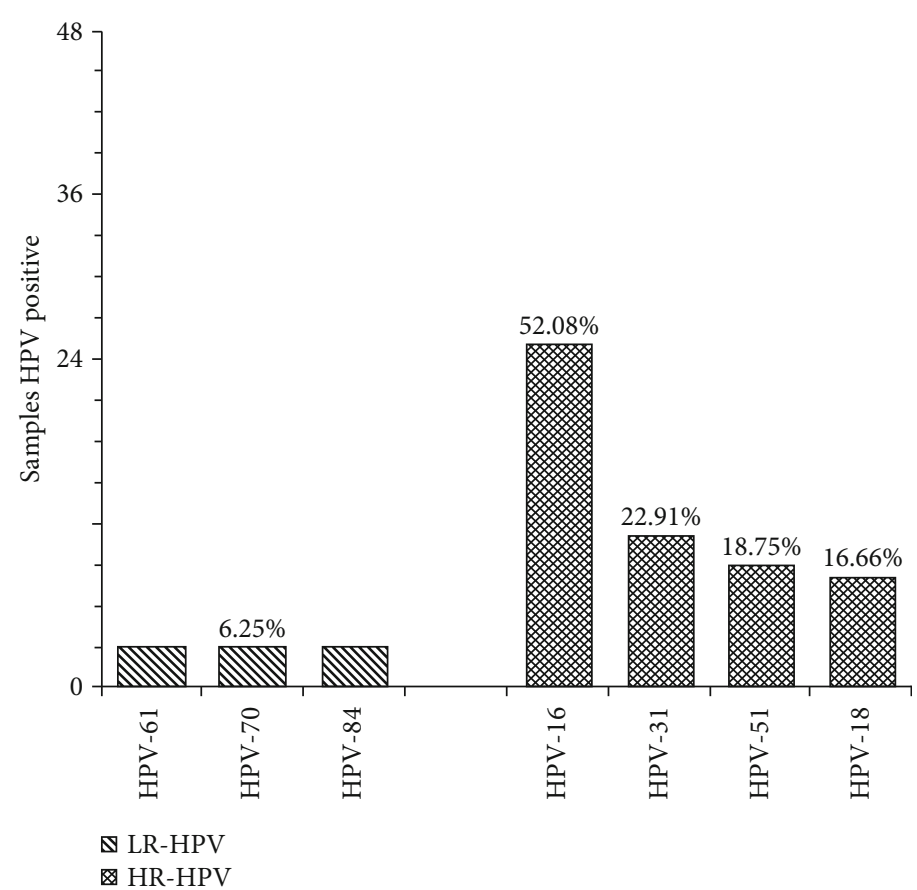

(b)

FIGURE 1: HPV groups present in samples with cervical lesion. The figure shows the number of positive samples by HPV group (a); moreover, subsection (b) depicts the number of positive samples by HPV genotype that was more present.

TABLE 1: HPV prevalence.

\begin{tabular}{lcccc}
\hline Samples HPV positive & HR-HPV positive & LR-HPV positive & HPV-18 positive & HPV-16 positive \\
\hline Single infection & 14 & 0 & 1 & 13 \\
Mixed coinfection & 19 & 19 & 5 & 5 \\
Simple coinfection & 13 & 2 & 8 & 7 \\
All samples HPV-positive $(n=48)$ & 46 & 21 & 8 & 25 \\
\hline
\end{tabular}

HPV-18 with other HR-HPV genotypes was detected in two samples (15.38\%) (Table 1). On the other hand, of the 19 samples with mixed coinfection, five (26.31\%) showed the presence of HPV-16, and five (26.31\%) showed the presence of HPV-18 (Table 1).

The OR statistical analysis revealed that coinfection with more than one HPV type has a probability of $97.6 \%$ of developing LSIL (Table 2). This estimate is considerably higher than those entertaining the likelihood of producing severe dysplasia, such as HSIL and CC (18.6\% and 5.8\%, respectively) (Table 2). On the other hand, we selected the three most prevalent HPV genotypes in our study (HPV-16, HPV-31, and HPV-51) to estimate their probability of developing a cervical lesion. OR analysis showed that the infection with HPV-31 and HPV-51 genotypes increases 5.5- and 7fold, respectively, the risk of developing LSIL, whereas infection with the HPV-16 genotype increases the risk of developing cervical cancer by 10.3 times (Table 3 ). In addition, the analysis of the 25 biopsies from adjacent regions in terms of cervical lesions without morphological changes demonstrated that $80 \%$ of these $(20 / 25)$ were HPV-positive. Inter- estingly, this percentage is very similar to that of HPVpositive biopsies with cervical lesion (87.27\%).

From these biopsies, all from patients with HSIL (10/10), $89 \%(8 / 9)$ from patients with CC, and 33\% (2/6) from patients with LSIL were HPV-positive. Although the percentages of HPV-positive samples were similar between cervical lesions and adjacent regions without morphological changes, the proportion of HPV coinfection was different. Samples without morphological changes but that were HPV-positive exhibited only HR-HPV genotypes with eight different HPV. All of these samples contained HPV-16 (Table 4); $70 \%(14 / 20)$ demonstrated a single infection, and 30\% (6/20) displayed coinfection (one sample with mixed coinfection and five with simple coinfection). Interestingly, all biopsies of the region adjacent to CC that were infected with HPV $(n=8)$ contained a single infection; while $40 \%$ (4/10) of biopsies without morphological changes from patients diagnosed with HSIL presented coinfection, and one of these showed four different HRHPV genotypes. Finally, the two biopsies without morphological changes from patients diagnosed with LSIL and who were HPV-infected revealed coinfection (Table 4). 
TABLE 2: Relationship between injury degree and coinfection.

\begin{tabular}{|c|c|c|c|c|}
\hline ASCUS & Single infection & Coinfection & $\mathrm{OR}=3.5000$ & Probability \\
\hline Lesion & 1 & 7 & 95\% CI (0.3884-31.5121) & \\
\hline Other cervical lesions & 13 & 27 & $\begin{array}{c}Z=1.117 \\
P=0.2641\end{array}$ & $* * *$ \\
\hline LSIL & Single infection & Coinfection & $\mathrm{OR}=41.0000$ & Probability \\
\hline Lesion & 0 & 20 & $95 \%$ CI (2.2597-743.9164) & \\
\hline Other cervical lesions & 14 & 14 & $\begin{array}{c}Z=2.511 \\
P=0.0120\end{array}$ & $97.6 \%$ \\
\hline HSIL & Single infection & Coinfection & $\mathrm{OR}=0.2299$ & Probability \\
\hline Lesion & 6 & 5 & 95\% CI (0.0555-0.9527) & \\
\hline Other cervical lesions & 8 & 29 & $\begin{array}{c}Z=2.027 \\
P=0.0427\end{array}$ & $18.6 \%$ \\
\hline $\mathrm{CC}$ & Single infection & Coinfection & $\mathrm{OR}=0.0625$ & Probability \\
\hline Lesion & 7 & 2 & $95 \%$ CI (0.0106-0.3675) & \\
\hline Other cervical lesions & 7 & 32 & $\begin{array}{l}Z=3.067 \\
P=0.0022\end{array}$ & $5.8 \%$ \\
\hline
\end{tabular}

TABLE 3: Relationship between of degree injury and HPV type.

\begin{tabular}{|c|c|c|c|c|c|}
\hline $\mathrm{CC}$ & Frequency & HPV-16 & Other HPV & $\mathrm{OR}=10.3529$ & Probability \\
\hline Lesion & & 8 & 1 & 95\% CI (1.1784-90.9562) & \\
\hline Other cervical lesions & $89.9 \%$ & 17 & 22 & $\begin{array}{l}Z=2.108 \\
P=0.0350\end{array}$ & $91 \%$ \\
\hline LSIL & Frequency & HPV-31 & Other HPV & $\mathrm{OR}=5.5556$ & Probability \\
\hline Lesion & & 8 & 12 & 95\% CI (1.2560-24.77) & \\
\hline Other cervical lesions & $40 \%$ & 3 & 25 & $\begin{array}{c}Z=2.248 \\
P=0.0245\end{array}$ & $85 \%$ \\
\hline LSIL & Frequency & HPV-51 & Other HPV & $\mathrm{OR}=7.0000$ & Probability \\
\hline Lesion & & 7 & 13 & 95\% CI (1.2702-38.5756) & \\
\hline Other cervical lesions & $35 \%$ & 2 & 26 & $\begin{array}{l}Z=2.235 \\
P=0.254\end{array}$ & $88 \%$ \\
\hline
\end{tabular}

Data obtained with the MedCalc ${ }^{\circledR}$ statistical program.

TABLE 4: HR-HPV prevalence and coinfection in HPV-positive samples without morphological changes areas adjacent to cervical lesions.

\begin{tabular}{|c|c|c|c|c|c|c|}
\hline $\begin{array}{l}\text { Areas without morphological } \\
\text { changes adjacent to: }\end{array}$ & $\begin{array}{c}\text { HR-HPV } \\
\text { positive ( } n=20 \\
\text { ) }\end{array}$ & $\begin{array}{l}\text { Single } \\
\text { infection }\end{array}$ & Coinfection & $\begin{array}{l}\text { HPV-16 } \\
\text { positive } \\
(n=20)\end{array}$ & $\begin{array}{c}\text { Two HR-HPV } \\
\text { genotypes }(n=6)\end{array}$ & $\begin{array}{l}\text { More than two HR-HPV } \\
\text { genotypes }(n=6)\end{array}$ \\
\hline LSIL $(n=2)$ & 2 & 0 & 2 & 2 & 2 & 0 \\
\hline HSIL $(n=10)$ & 10 & 6 & 4 & 10 & 3 & 1 \\
\hline $\mathrm{CC}(n=8)$ & 8 & 8 & 0 & 8 & 0 & 0 \\
\hline Total $n(\%)$ & $20(100 \%)$ & $14(70 \%)$ & $6(30 \%)$ & $20(100 \%)$ & $3(83.33 \%)$ & $1(16.66 \%)$ \\
\hline
\end{tabular}

In summary, our data demonstrated a high prevalence of multiple HPV-types infection in Mexican patients with different grades of cervical lesion, a condition that could participate in the development of the diverse injuries.

\section{Discussion}

Cervical cancer is the fourth most frequent neoplasm worldwide, affecting 570,000 women and causing more than 311,000 deaths in the last year [14]. In Mexico, CC is the third most prevalent cancer in women, with 7,689 new cases reported in 2018 [15]. Although HPV is the causal agent for the development of cervical precancerous lesions and CC, the role of infections with different types of HPV or coinfections remains unclear.

Here, we analyzed the presence of HPV single infection and coinfection in Mexican patients with different cervical lesions. Our data showed an 87.2\% HPV detection rate in these samples, while previous studies reported rates as high as $98 \%$ [16], and the global prevalence of HPV infection in 
women is $65 \%$ [17]. The disparity of these data with our results could perhaps be explained by the size of our sample $(n=55)$. Moreover, the percentage of negativity $(12.73 \%)$ in our samples could be related to the PCR type employed (simple PCR with primers MY09/MY11). In addition, we cannot rule out that negative cases in our study may be applicable to viral load, which could be below the severity of the technique. Other studies have concluded that utilizing a nested PCR using the MY09/MY11 and GP5+/GP6+ primers can significantly increase the sensitivity from 10 to 100 times, compared with the simple PCR employing only the MY09/MY11 primers [12]. In this respect, a multicenter study in Spain and Colombia using the MY09/MY11 primers identified viral DNA in $75 \%$ of cases; the rest of the samples were reanalyzed using the nested PCR system and achieved a $20 \%$ increase in the range of detection [18].

Nevertheless, we found an HPV incidence greater than or equal to $80 \%$, regardless of the injury type diagnosed. However, we did not detect a correlation between the presence of infection and lesion type $(P=0.235)$. The data suggest that, although HPV infection is involved in the development of cervical lesions, it is not sufficient to predict the injury type that could be produced. In agreement with previous reports, these results suggest that other factors might be implicated in the development of each lesion type, including the viral genotype and the presence of more than one viral type [19]. The genotypes distribution found in our study is similar to that previously reported in Mexico [20]. We found that HPV-16 and HPV-18 are the most common genotypes, which is consistent with previous studies carried out with Mexican patients [17, 21]. Other HPV types detected were HPV-31, HPV-51, HPV-18, HPV-58, and HPV-53, which are the most prevalent in different states of the Mexican Republic [22-26]. However, worldwide, there are geographic differences in the frequency of HPV types; for instance, HPV-52 and HPV-58 were the most prevalent types in HSIL and $\mathrm{CC}$ in China $[27,28]$. Indeed, these genotypes are more common than HPV-43, HPV-31, and HPV-33 throughout Asia [27], whereas in Mali, Africa, HPV-51 and HPV-73 are more frequent than HPV-16 and HPV-18 [21].

Sasagawa et al. [28] found, according to their OR analysis, that HPV-11, HPV-39, HPV-42, HPV-44, HPV-53, HPV-59, HPV-62, and HPV-66 were associated with LSIL, HPV-33, HPV-35, and HPV-56 that were associated to HSIL, while HPV-16, HPV-18, HPV-31, HPV-51, HPV-52, and HPV58 were associated to invasive carcinoma. In our study, the most common genotypes in LSIL were HPV-31 (40\%) and HPV-51 (35\%), which are risk factors for developing this injury ( $\mathrm{OR}=5.5$ and $\mathrm{OR}=7$, respectively). In the same manner, HPV-16, the most frequent genotype in patients diagnosed with cervical cancer, increased by 10.3 times the likelihood of developing this lesion. However, we utilized a low-density microarray to detect the 35 viral genotypes most prevalent worldwide; therefore, viral types undetectable by this microarray could be present in our samples.

On the other hand, we observed a frequency of coinfection of $70.83 \%$, which is consistent with previous reports. For instance, García-Espinosa et al. [29] reported a coinfection of $78.8 \%$ in Equatorial Guinea. Another study conducted in Japanese women associated multiple HPV infections with LSIL, HSIL, and CC in 95\% of samples, where in which the prevalence decreased with the degree of the injury [28]. Concerning coinfections, it has been suggested that a synergism exists among the coinfecting HPV genotypes, or contrariwise, different HPV types could compete to colonize the cervical epithelium [10].

Infection with multiple HPV-oncogenic types is prevalent, especially among younger women [30]. The effects of the infection with multiple HPV types on the risk of developing precancerous lesions and cancer are not well understood. In general, it has been assumed that each HPV genotype acts independently; thus, each should contribute individually to the risk of the progress of cytological and precancerous changes [10]. To support this idea, it has been observed that individual histological cervical lesions are usually caused by a single genotype; although, several types are present in the cytology [31]. Under this assumption, we would expect that infection with multiple HPV types produces additive effects to the risk of having abnormal cytology and precancerous cervical lesions. Interestingly, in our samples, we simultaneously detected HR- and LR-HPV, and particularly, LRHPV were not found alone but were always detected together with HR-HPV genotypes. In our population, we detected six HPV genotypes (HPV-16, HPV-18, HPV-31, HPV-56, HPV66 , and HPV-6) in the biopsy of a patient diagnosed with HSIL. Five of these genotypes are HR-HPVs and one LRHPV. According to the literature $[8,9,28]$, it is likely that, in this case, there is the presence of LR-HPV, entertaining an association with a HR-HPV that may lead to duplication of viral charges and exert a synergistic effect in the oncogenesis.

In this study, we found that coinfection correlated with the injury type, supporting previous reports that afford value to the detection and characterization of multiple HPV infections in clinical samples in order to search for markers for abnormal cervical cells.

Studies of HPV coinfection in Mexico are scarce to date; Aguilar-Lemarroy et al. [32] reported that, in samples diagnosed with CIN-I and CIN-III, the percentage of coinfection was 58.2 and $60 \%$, respectively, whereas the incidence of coinfection in cervical cancer decreased to $26.4 \%$. Similarly, we found a higher percentage of coinfection in LSIL (100\%) and ASCUS (88\%) than in HSIL and CC (45\% and $22 \%$, respectively). Thus, the presence of coinfection increased, to a greater extent, the chance of developing LSIL (OR $=41, P=0.02)$ than HSIL $(\mathrm{OR}=18.6, P=0.05)$ or cervical cancer $(\mathrm{OR}=5.6, P=0.05)$. This result supports the hypothesis that the subsequent accumulation of different infections can be a marker of persistent infection and, eventually, a possible risk factor for the progression of the lesion.

Another key factor in the development of cervical lesions is the presence of simple or mixed coinfections [3]. We observed a high frequency of mixed coinfections (HR-HPV + LR-HPV) in LSIL and ASCUS (70\% and 65\%, respectively) and severe injuries such as HSIL and cervical cancer, and a higher frequency of simple coinfections (HR-HPV $+\mathrm{HR}$ HPV) (100\% and 80\%, respectively). 
It is noteworthy that $36 \%$ of the observed coinfection contains HPV-16; similarly, López-Rivera et al. [20] reported that this viral genotype is present in $43 \%$ of the coinfected population. Despite this, we observed high single infections containing HPV-16 (13/25 HPV-16 positive). Another important datum of our work is that HPV-31 was found only in coinfections.

Regarding results about the adjacent regions without morphological changes, the prevalence of HPV infection $(80 \%)$ was similar to that found in lesion biopsies. However, coinfection was low (30\%) in the adjacent areas without morphological changes of patients with cervical lesions; although, all of these HPV-positive areas could develop some injury over time, considering that the prevalence of HPV-16 in these regions was $100 \%$. Bearing these data and the OR results $(\mathrm{HPV}-16 / \mathrm{CC}, \mathrm{OR}=10.3, P \leq 0.05)$ in mind, we were able to predict that all these regions have a $91 \%$ chance of developing cervical cancer. It is noteworthy that these results are not comparable with those found in the literature, because majority of studies only genotypify the HPV found in intraepithelial injuries, but not in the areas adjacent to the cervical lesion. In this regard, it is important to note that these results highlighted the importance of studies about viral genotyping, even if patients do not exhibit any injury by colposcopy, as it would aid in timely diagnosis and appropriate treatment for the prevention of the development of intraepithelial lesions and cervical cancer. In recent years, several epidemiological studies have mentioned the presence of single infections and coinfections in control groups. For instance, Aguilar-Lemarroy [32] reported that $68.8 \%$ of healthy controls are infected with HPV-16 and a high percentage of coinfections with other HPV. These data and our results demonstrate that, in addition to HPV-16 (the most prevalent HPV worldwide), it is necessary to pay attention to coinfection in conjunction with genotypes that, although are less frequent, they may entertain local epidemiological importance. The high frequency of HR-HPV in cases of LSIL and HSIL emerges as a reasonable explanation for the high incidence of cervical cancer in our region. Also, determining the frequency and distribution of HPV genotypes can help to measure the effectiveness of an eventual vaccine in our community and aid in streamlining the resources of our national cervical cancer-screening program.

In summary, our data demonstrate the incidence of multiple HPV-type infections in the different grades of the cervical lesion. These data may help to develop better clinical strategies against cervical lesions caused by HPV coinfections and in the search for a more efficient treatment.

\section{Conclusions}

A total of $87.27 \%$ of Mexican patients with cervical lesions studied here demonstrated HPV infection, and the most frequent genotypes were HPV-16, HPV-31, HPV-51, and HPV18. The prevalence of HPV coinfection in the cervical lesions was $70.83 \%$, and coinfection with certain types correlates with the type of cervical lesion, determining that these factors can be used as a predictor of the degree of the injury; in particular, HPV-16 increases the chance to develop cervical can- cer. Moreover, HPV detection in healthy areas could be a predictor for the development of injury, given that prevalence of infection in these regions was $80 \%$.

\section{Data Availability}

All data generated of analysis during this study are included in this published article.

\section{Ethical Approval}

This study was approved by the Committee of Bioethics of Hospital Juárez de México. (Approval number: HJM 2231/13-B).

\section{Consent}

Informed consent was obtained from the patient for publication of this paper.

\section{Conflicts of Interest}

The authors have declared no conflict of interest.

\section{Authors' Contributions}

All authors read and approved the final manuscript.

\section{Acknowledgments}

We are sincerely grateful to the subjects who participated in this research. We also thank Dra. Fabiola Fragozo-Sandoval and Mónica Sierra-Martínez of Hospital Juárez de México (Mexico City), the Oncology and Pathology Departments of Hospital La Raza (Mexico City); and Jaime Ignacio Bárcenas-Trejo of the Centro de Salud Nueva Atzacoalco, Secretaría de Salud de la Ciudad de México (Mexico City). This work was supported by the Consejo Nacional de Ciencia y Tecnología (CONACyT-México) (grant CB-2012/176983 to López-Reyes I). Padilla-Mendoza, JR, received a Doctoral scholarship from CONACyT (705048). The funders played no role in study design, data collection, and analysis, decision to publish, or preparation.

\section{References}

[1] H. zur Hausen, "Papillomaviruses in the causation of human cancers - a brief historical account," Virology, vol. 384, no. 2, pp. 260-265, 2009.

[2] V. Granados-García, Y. N. Flores, R. Pérez, S. E. Rudolph, E. Lazcano-Ponce, and J. Salmerón, "Cost of the cervical cancer screening program at the Mexican Social Security Institute," Salud Pública de México, vol. 56, no. 5, pp. 502-510, 2014.

[3] A. Gradíssimo and R. D. Burk, "Molecular tests potentially improving HPV screening and genotyping for cervical cancer prevention," Expert Review of Molecular Diagnostics, vol. 17, no. 4, pp. 379-391, 2017.

[4] S. M. A. Kulmala, I. P. Shabalova, N. Petrovitchev et al., "Typespecific persistence of high-risk human papillomavirus infections in the New Independent States of the former Soviet 
Union Cohort Study," Cancer Epidemiology Biomarkers \& Prevention, vol. 16, no. 1, pp. 17-22, 2007.

[5] K. Matsumoto, A. Oki, R. Furuta et al., "Predicting the progression of cervical precursor lesions by human papillomavirus genotyping: a prospective cohort study," International Journal of Cancer, vol. 128, no. 12, pp. 2898-2910, 2011.

[6] P. Brianti, E. De Flammineis, and S. R. Mercuri, "Review of HPV-related diseases and cancers," The New Microbiologica, vol. 40, no. 2, pp. 80-85, 2017.

[7] D. Bzhalava, C. Eklund, and J. Dillner, "International standardization and classification of human papillomavirus types," Virology, vol. 476, pp. 341-344, 2015.

[8] L. De Brot, B. Pellegrini, S. T. Moretti et al., "Infections with multiple high-risk HPV types are associated with high-grade and persistent low-grade intraepithelial lesions of the cervix," Cancer Cytopathology, vol. 125, no. 2, pp. 138-143, 2017.

[9] A. Carrillo-García, S. Ponce-de-León-Rosales, D. Cantú-deLeón et al., "Impact of human papillomavirus coinfections on the risk of high-grade squamous intraepithelial lesion and cervical cancer," Gynecologic Oncology, vol. 134, no. 3, pp. 534-539, 2014.

[10] P. A. Orlando, R. A. Gatenby, A. R. Giuliano, and J. S. Brown, "Evolutionary ecology of human papillomavirus: trade-offs, coexistence, and origins of high-risk and low-risk types," The Journal of Infectious Diseases, vol. 205, no. 2, pp. 272-279, 2012.

[11] J. L. Benedet, H. Bender, Jones H 3rd, H. Y. Ngan, and S. Pecorelli, "FIGO staging classifications and clinical practice guidelines in the management of gynecologic cancers. FIGO Committee on Gynecologic Oncology," International Journal of Gynaecology and Obstetrics: The Official Organ of the International Federation of Gynaecology and Obstetrics, vol. 70, no. 2, pp. 209-262, 2000.

[12] M. Camargo, S. Soto-De Leon, R. Sanchez et al., "Detection by PCR of human papillomavirus in Colombia: comparison of GP5+/6+ and MY09/11 primer sets," Journal of Virological Methods, vol. 178, no. 1-2, pp. 68-74, 2011.

[13] Y. J. Choi and J. S. Park, "Clinical significance of human papillomavirus genotyping," Journal of Gynecologic Oncology, vol. 27, no. 2, pp. e21-e21, 2016.

[14] F. Bray, J. Ferlay, I. Soerjomataram, R. L. Siegel, L. A. Torre, and A. Jemal, "Global cancer statistics 2018: GLOBOCAN estimates of incidence and mortality worldwide for 36 cancers in 185 countries," CA: a Cancer Journal for Clinicians, vol. 68, no. 6, pp. 394-424, 2018.

[15] E. Hurtado-Salgado, E. Ortiz-Panozo, J. Salmerón et al., "Use of HPV testing in cervical cancer screening services in Mexico, 2008-2018: a nationwide database study," Salud Publica de Mexico, vol. 60, no. 6, pp. 722-733, 2018.

[16] A. Melo, I. Roa, S. Montenegro, I. Capurro, and J. C. Roa, "Estudio comparativo de detección del virus papiloma humano (VPH) en muestras citológicas y biopsias de cuello uterino," Revista Médica de Chile, vol. 133, no. 6, pp. 639644, 2005.

[17] E. Lazcano-Ponce, R. Herrero, N. Muñoz et al., "Epidemiology of HPV infection among Mexican women with normal cervical cytology," International Journal of Cancer, vol. 91, no. 3, pp. 412-420, 2001.

[18] K. Sotlar, D. Diemer, A. Dethleffs et al., "Detection and typing of human papillomavirus by e6 nested multiplex PCR," Journal of Clinical Microbiology, vol. 42, no. 7, pp. 3176-3184, 2004.
[19] P. Tsikouras, S. Zervoudis, B. Manav et al., "Cervical cancer: screening, diagnosis and staging," Journal of BUON, vol. 21, no. 2, pp. 320-325, 2016.

[20] M. G. López Rivera, M. O. Flores, J. D. Villalba Magdaleno, and V. Sánchez Monroy, "Prevalence of human papillomavirus in women from Mexico City," Infectious Diseases in Obstetrics and Gynecology, vol. 2012, Article ID 384758, 4 pages, 2012.

[21] N. Muñoz, F. X. Bosch, S. de Sanjosé et al., "Epidemiologic classification of human papillomavirus types associated with cervical cancer," The New England Journal of Medicine, vol. 348, no. 6, pp. 518-527, 2003.

[22] L. F. Sánchez-Anguiano, C. Alvarado-Esquivel, M. A. ReyesRomero, and M. Carrera-Rodríguez, "Human papillomavirus infections in women seeking cervical Papanicolaou cytology of Durango, Mexico: prevalence and genotypes," BMC Infectious Diseases, vol. 6, no. 1, p. 27, 2006.

[23] B. Illades-Aguiar, E. M. Cortés-Malagón, V. Antonio-Véjar et al., "Cervical carcinoma in Southern Mexico: human papillomavirus and cofactors," Cancer Detection and Prevention, vol. 32, no. 4, pp. 300-307, 2009.

[24] N. Velázquez-Márquez, M. A. Paredes-Tello, H. Pérez-Terrón, G. Santos-López, J. Reyes-Leyva, and V. Vallejo-Ruiz, "Prevalence of human papillomavirus genotypes in women from a rural region of Puebla, Mexico," International Journal of Infectious Diseases, vol. 13, no. 6, pp. 690-695, 2009.

[25] B. Illades-Aguiar, L. C. Alarcón-Romero, V. Antonio-Véjar et al., "Prevalence and distribution of human papillomavirus types in cervical cancer, squamous intraepithelial lesions, and with no intraepithelial lesions in women from Southern Mexico," Gynecologic Oncology, vol. 117, no. 2, pp. 291-296, 2010.

[26] A. Orozco-Colín, A. Carrillo-García, A. Méndez-Tenorio et al., "Geographical variation in human papillomavirus prevalence in Mexican women with normal cytology," International Journal of Infectious Diseases, vol. 14, no. 12, pp. e1082-e1087, 2010.

[27] Y. Liu, Y. Pan, W. Gao, Y. Ke, and Z. Lu, "Whole-genome analysis of human papillomavirus types 16, 18, and 58 isolated from cervical Precancer and Cancer samples in Chinese women," Scientific Reports, vol. 7, no. 1, p. 263, 2017.

[28] T. Sasagawa, W. Basha, H. Yamazaki, and M. Inoue, "Highrisk and multiple human papillomavirus infections associated with cervical abnormalities in Japanese women," Cancer Epidemiology, Biomarkers \& Prevention : A Publication of The American Association for Cancer Research, Cosponsored by the American Society of Preventive Oncology, vol. 10, no. 1, pp. 45-52, 2001.

[29] B. García-Espinosa, M. P. Nieto-Bona, S. Rueda et al., "Genotype distribution of cervical human papillomavirus DNA in women with cervical lesions in Bioko, Equatorial Guinea," Diagnostic Pathology, vol. 4, no. 1, p. 31, 2009.

[30] K. Syrjänen, "New concepts on risk factors of HPV and novel screening strategies for cervical cancer precursors," European Journal of Gynaecological Oncology, vol. 29, no. 3, pp. 205221, 2008

[31] F. X. Bosch, A. Lorincz, N. Munoz, C. J. L. M. Meijer, and K. V. Shah, "The causal relation between human papillomavirus and cervical cancer," Journal of Clinical Pathology, vol. 55, no. 4, pp. 244-265, 2002.

[32] A. Aguilar-Lemarroy, V. Vallejo-Ruiz, E. I. Cortés-Gutiérrez et al., "Human papillomavirus infections in Mexican women with normal cytology, precancerous lesions, and cervical cancer: type-specific prevalence and HPV coinfections," Journal of Medical Virology, vol. 87, no. 5, pp. 871-884, 2015. 